\title{
Research on Application of Large Data Processing Technology in Interactive Design
}

\author{
Li Chunxiao, Zhou Xiaojuan \\ Xi' an international university , Institute of technology, Department of computer science and \\ technology, shanxi Xi 'an 710077
}

Keywords: Application, Large Data Processing Technology, Interactive Design

\begin{abstract}
Interactive design is a kind of technology which can realize the two-way interaction between man and machine. It is widely used in product design and so on, but it is weak in data processing. Therefore, it will apply the large data processing technology in interactive design Situation to explore and discuss.
\end{abstract}

\section{Introduction}

With the development of computer technology, the Internet every day to produce a lot of data, the data has changed from quantitative to qualitative change, as a "big data" . It has entered the field of bioinformatics, weather forecasting and other fields. In the era of large data, the user's preferences, behavior and other data, such as the Internet habits, browsing traces, participation in the topic and the contents of the comments have been made public on the Internet, and have a large scale. This information will change the traditional interactive design methods, such as no longer through the user interviews, questionnaires in order to obtain user habits, and the traditional method of investigation of individual users to get the results far from large data statistical analysis of the results of a comprehensive and persuasive.

Interactive design covers a wide range of knowledge, a wide range of applications, in order to meet people's changing material needs, spiritual needs, designers need to analyze a large number of user data, but a single interactive design cannot make a lot of reliable data analysis And judgment, large data processing technology can make up for its shortcomings, therefore, this technology is widely used in interactive design is of great practical significance, to explore this is necessary.

\section{The Research Status of Large Data Processing}

From 1960 to early 1980, many companies used key applications such as finance and banking on large-scale mainframes. Storage media included tapes, disks, and discs that were not commonly used. At that time, these were called big data, but at that time the big data and the current large data compared to the great gap. With the emergence of PC and the application of the emergence of a lot of internal office documents as the main form of data, the main form of documents, forms and presentations, as well as later photos, videos and music. The company's financial staff and a large number of office workers are at this time to produce large amounts of data, and quickly promote the growth of data. The third large-scale growth of data comes with the rise of the Internet. At the same time, QQ, micro-blogging, We-Chat, multimedia applications will take the initiative to generate data, search engines and web browsing, etc. will passively record data. And now, the Internet, Internet of Things, cloud computing and other emerging applications mature application, so that the data processing was explosive exponential growth, the enterprise data also reached an unprecedented level of pet. At present, experts at home and abroad on the progress of large data research is only reached a consensus on the scale, for example, "super-scale" that is GB-level data, "massive" that is terabyte data, and large data that PB Level above the data.

Big data now permeates all areas of people's lives. The rapid development of large data so that interactive technology has also undergone a corresponding change and now interactive design technology to the user's habits, personal hobbies and interests and the information published on the 
Internet. Compared to the past few decades, now this technology has formed a certain scale. In the past, many of the cumbersome steps to collect information can be omitted, and their own use of large data to analyze the data can be analyzed very comprehensive and very detailed. Of course, the technology is not very perfect, but also need to further solve the machine learning, model learning and other auxiliary learning problems.

\section{The Application System Framework of Large Data Processing Technology in the Interactive Design}

Human-computer interaction efficiency and nature are one of the key factors influencing its development. First of all, large data in the process of dealing with human-computer interaction in the process of bulk processing of the system, the system is the process of the Internet generated by the massive non-transferable data processing. Such as the user in the site click-through rate and the number of pages and other data processing, to determine which customers prefer a product. Google's large-scale distributed file system and large-scale distributed system is a typical and useful batch data processing system. The system for streaming data is a system for real-time processing of large amounts of online data present in the Internet. These online data have complex formats that are not available in other data, and the data is continually derived from a number of different channels that require efficient and rapid processing of these real-time online data. Such as animal and human organisms in the sensor data, shopping malls in the flow of data, positioning systems and other data need to efficiently and orderly real-time processing. Typical streaming data processing systems include Storm systems. An interactive data processing system is an emerging system that can process data in a human-computer interaction. How to improve the speed of universal computing, improve the efficiency of human-computer interaction, to achieve the expansion of information space interaction needs further study.

From the stage of interactive design, large data processing technology is mainly used in testing and user research of these two links. Specifically, the system framework consists of three parts. The first is the user research and this stage of the work is entirely based on large data processing technology. First, the system can automatically obtain valid data, the application of crawler technology to achieve access to information automation. Second, after the data collection technology to be necessary to analyze and deal with the data, this time used in the technology for distributed and parallel processing technology, even if the amount of data and data scale, the technology is also more applicable. Then, the system can be through data mining, machine learning way to start automated learning, so that designers understand the user's personal habits and preferences. The second is the version update. This stage of the work of the basic A / B test, when the system software is updated, the designer can be random to the user to push the old and new versions, and then conversion rate, traffic and other data collection, the data after a comprehensive collection. The designer can compare the functions of the two versions, analyze the respective advantages of the old and new versions, and then optimize the version update scheme. The third is user experience optimization. The work of this stage is based on pattern recognition. First, the designer can collect the data information with the support of the computer technology. Secondly, the designers can use the information visualization and large data processing technology to analyze the original user data, and through the pattern recognition technology Finally, the designers according to the relevant data on the user's habits of analysis and summary, and the interface and system functions to adjust and optimize the information to form a mental model, more in line with the actual needs of users.

\section{The Interaction Design at Large Data Age}

This paper analyzes and predicts the development trend, interactive design method, process, model, and evaluation of large interactive design in the era of large data information, in view of the connotation and extension of large interactive design of large data information age, the development process and the research content and scope. Through the analysis of large data analysis and user research, PETSC (political, economic, technical, social and cultural), and interactive design in the 
field of network, mobile equipment and games, the non-causality, discontinuity, uncertainty, volatility, unpredictability and the principle of ecosystem reference system. This paper expounds the four roles of interaction design in the strategic layer, the scope layer, the structure layer, the framework layer and the presentation layer, and puts forward the use of Newton thinking and quantum thinking to combine the information thinking, combined with the interaction of cognitive logic and information cognitive emotion Meaning as the center "of the ecological sustainable large interactive design method.

This paper analyzes and predicts the development trend, interactive design method, process, model, and evaluation of large interactive design in the era of large data information, in view of the connotation and extension of large interactive design of large data information age, the development process and the research content and scope. Through the analysis of large data analysis and user research, PETSC (political, economic, technical, social and cultural), and interactive design in the field of network, mobile equipment and games, the non-causality, discontinuity, Uncertainty, volatility, unpredictability and the principle of ecosystem reference system. This paper expounds the four roles of interaction design in the strategic layer, the scope layer, the structure layer, the framework layer and the presentation layer, and puts forward the use of Newton thinking and quantum thinking to combine the information thinking, combined with the interaction of cognitive logic and information cognitive emotion Meaning as the center "of the ecological sustainable large interactive design method.

Interactive design is the design of the design of software, mobile devices, artificial environments, services, wearable devices and other artificial systems. Before the design, we must first understand the user's psychology usually needs to conduct a survey, access to user needs. Traditional methods of investigation include observing, interviewing and questionnaires. Observe the law is in the natural conditions, the psychological activities of the external activities of a systematic, planned observation, from which to find the psychological phenomenon of the emergence and development of the law. Interviews are a way of understanding the motivations, attitudes, personality, and values of the interviewer through interviews between the interviewer and the interviewee. Questionnaires are prepared in advance to understand the issues listed in the questionnaire so that consumers answer, through the analysis of the answer and statistical studies, draw the appropriate conclusions of the method. These methods are based on observation and then artificially summarize user needs.

\section{The Update Based on the A / B Version Test}

In the use of A / B test before the need to create a new test page, the title of the font, the background color, wording, etc. in this test page with the original page are very different, and then the two pages will be random Draw the way at the same time push to all online browsing users. The next two pages of user conversion rate will be counted at the same time and the user can clearly understand the advantages and disadvantages of the two designs. The traditional A / B test is a way to randomly assign each group of variables to a specific single variable processing level, compare the performance of multiple test groups with the control group, and test the way. The new A / B test does not simply limit its scope to Web analytics, but rather injects fresh blood and life into it, ie mobile device side analysis. Co-founder and CEO Adam Ceresko said that today, the development of science and technology personnel must greatly improve the performance of optimization tools, mobile analysis has become the fastest and most developed A / B test of a new area.

\section{The User Research under the Big Data}

The first use of web crawler technology to automatically obtain the effective data on the Internet; and then use the distributed and parallel processing technology to collect the massive data processing; finally, the use of machine learning, data mining methods automatically learn and get user preferences.

To web design, for example, the first use of web crawler technology automatically access different visits to the flow of the site, such as access to large traffic large portal, similar to Sohu, Sina, etc., as 
well as access to the flow of small professional site, Website structure and content, such as text, pictures and so on. Web crawler technology is a process in accordance with certain rules and automatically crawl the World Wide Web information or script.

And then use the distributed and parallel processing technology to collect the massive data processing, sort out the site design of different concerns of the data, such as site layout, color matching, link relationship. Distributed processing and parallel processing are two different architectures that are used to improve the speed of computer processing. Parallel processing is the use of multiple functional components or multiple processors work together to improve system performance or reliability; distributed processing is different locations, or has different functions, or has different data of multiple units

The computer is connected through the communication network, under the unified management of the control system, the coordination of large-scale information processing tasks. Finally, the use of machine learning, data mining methods automatically learn and get user preferences, get the site layout, color matching, link relationship. The main research method of large data is the data mining in computer science, and its basic goal has two, namely, description and prediction. Describe the valuable models and laws in the data by describing the potential patterns in the massive data and predicting them based on the underlying patterns in the data. Based on large data processing technology, finishing into the human can interpret the form of information.

\section{Conclusion}

With the continuous development of society, science is also progressing, which makes large data processing technology in the interactive design to be more widely used, the use of large data processing technology to make people's lives more convenient. Large data processing technology not only provides us with strong data support and solid theoretical basis for our traditional user research and new test methods in design, but also greatly reduces labor costs, and further for the design and computer science crossed the exploration, and provided useful theories and methods.

\section{References}

[1] Chen Zhigang, Lu Xiaobo. The change and development of information and interaction design under the background of large data [J]. Packaging Engineering. 2015 (08)

[2] Tan Jingyan. Large interactive design of large data age[J]. Packaging Engineering. 2015 (08)

[3] Hu Zhongyan, Cao Yang, Sun Jianhua.Application of Pattern Recognition Technology in Concept Design of Automatic Classification Trash [J]. Packaging Engineering. 2008 (12)

[4] Lu Xinhua, Sun Jigui, Han Xiao Song, Xing Rong. Artificial life: the forefront of computer and biology of the cutting-edge disciplines [J] .Computer Science. 2008 (05)

[5] Li Yufeng, Huang Yiping.Study on Quantitative and Rational Method of Color Planning [J]. Packaging Engineering. 2004 (06)

[6] Ni Jun, Liu Hua. Computational physics frontier and its intersection with computing technology [J]. Physics. 2002 (07)

[7] Wang Xunkun. Interdisciplinary, cross science and its status in the scientific system[J]. Natural dialectics research. $2000(01)$ 